\title{
Not unreasonable: Why two negatives don't make a positive
}

\author{
Michael Henry Tessler ${ }^{1,3} \&$ Michael Franke ${ }^{2}$ \\ ${ }^{1}$ Massachusetts Institute of Technology, Department of Brain and Cognitive Sciences \\ ${ }^{2}$ University of Osnabrück, Department of Cognitive Sciences \\ ${ }^{3}$ Stanford University, Department of Psychology
}

\begin{abstract}
Logic tells us that two negatives make a positive, but in language, things are not so black and white: A person not unhappy may not be entirely happy. We hypothesize that innovative uses of double negatives like not unhappy stem from listeners entertaining flexible meanings for negation markers like not and un-, which context can then help disambiguate. We formalize this hypothesis in a computational model of language understanding, which predicts that not unhappy means something different than happy but also makes the additional prediction that single negations (unhappy vs. not happy) are interpreted identically except when a speaker uses both in the same context, which we confirm experimentally. Even double negations that flagrantly use the same negation marker twice (e.g., not not happy) are interpreted in subtle ways. These findings suggest that even one of the most logical elements of language - negation - can mean many things at once.
\end{abstract}

Keywords: semantics; pragmatics; negation; Bayesian cognitive model; Rational Speech Act

This work was supported in part by NSF Graduate Research Fellowship DGE-114747 to MHT.

Correspondence concerning this article should be addressed to Michael Henry Tessler, 43 Vassar St, Cambridge, MA 02139. E-mail: tessler@mit.edu 
I've been waiting for you all week. Don't you know that it is rotten time to not not be me? (El-P, "Works every time (feat. Paul Banks)")

Banal statements are given an appearance of profundity by means of the "not un-" formation. [...] It should be possible to laugh the "not un-" formation out of existence by memorizing this sentence: "A not unblack dog was chasing a not unsmall rabbit across a not ungreen field." (Orwell, 1946) (p. 357)

\section{Introduction}

Language is used to convey our thoughts, but we think and feel in ways that are not always easily expressible in words. Occasionally, speakers will coin a new term to express a subtle feeling, like being "plateaued" (Bardwick, 1986) or residing in a "zone of indifference" (Sapir, 1944). But we also use the tools already available to us, at the time we want to convey our message, to navigate and communicate the gradations of our thoughts and feelings (e.g., through metaphoric language; Lakoff, 2008).

Compositionality enables productive language use, and double negations are a classic example of using simple linguistic tools to convey subtle meanings. If Mary is not unhappy, does that mean she is happy? It doesn't seem exactly right:

$[\mathrm{T}]$ wo negatives do not exactly cancel one another [...]; the longer expression is always weaker: "this is not unknown to me" or "I am not ignorant of this" means "I am to some extent aware of it," etc. (Jespersen, 1924) (p. 332)

In other words, not unhappy (a negated antonym) should indicate a slightly positive state, below that of happy but perhaps more positive than neutral (i.e., above the zone of indifference; Sapir, 1944). The intuitions are subtle, not universally agreed upon (see Orwell's 1946 quote above), and further complicated by the fact that the meanings of not happy and unhappy are not obviously different from one another. Some argue that the two expressions have the same meaning (e.g., Jespersen, 1917; Blutner, 2004), while others disagree (e.g., Krifka, 2007), citing examples like:

It's an absolutely horrible feeling to be unhappy, and I don't even think I was unhappy, just not happy, if you know what I mean.

The interpretation of double negatives is interesting from both a logical perspective (Horn, 1989; Krifka, 2007) as well as a legal one, where double negations are surprisingly common (Tiersma, 1999). Also, insofar as novel meanings can be expressed through the iterative application of the same logical operation (i.e., two negatives), understanding this language could provide a window into richer conceptual spaces like emotions (Lindquist, Satpute, \& Gendron, 2015; Satpute et al., 2016), where negation has been shown to modulate physiological responses (Herbert, Deutsch, Sütterlin, Kübler, \& Pauli, 2011).

Theorizing about the meaning of negation goes back to Aristotle, who noted that there are several opposites. Contradictory opposites must have opposite truth values, e.g., "No humans are rational" and "Some humans are rational". Contrary opposites cannot both 

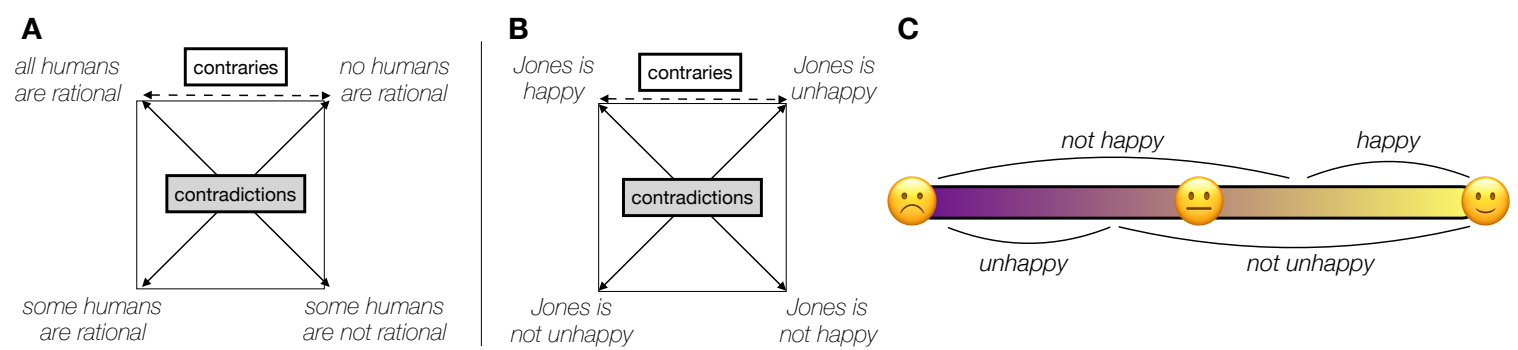

Figure 1. Contraries and contradictions as two different kinds of negation. A: Aristotle's classic square of opposition applied to quantifiers (all, some, none). B: An Aristotelean analysis of negated antonyms (happy, unhappy, not unhappy). C: Interpretations of negated antonyms in terms of degrees of happiness under an Aristotelean analysis.

be true, but can both be false, e.g., "All humans are rational" and "No humans are rational" (Figure 1A).

How should the negation markers not and un- (used here as a stand-in for morphological negation markers like in-, im-, dis-, etc.) map onto the different kinds of negation? An Aristotelean hypothesis would assign one to be a contradictory opposite and another to be a contrary (Figure 1B); a double negative (not unhappy) in this analysis would convey a contradiction of a contrary, resulting in a meaning distinct from that of the positive adjective (happy), while also enforcing that the contrary (unhappy) has a more negative meaning than the contradiction (not happy; Figure 1C; Horn, 1991; Krifka, 2007). An Orwellian analysis, on the other hand, only acknowledges contradictory opposition as an interpretation for negation markers; unhappy and not happy have the same interpretation, and doubles-negatives are posited to be entirely redundant (Figure 2; Orwell, 1946). ${ }^{1}$

We propose a third, hybrid alternative: the logical distinction between contradictory vs. contrary opposition creates an ambiguity in the meanings negation markers. This Flexible Negation model predicts that listeners interpret double negatives as distinct from simple positives not because the negation markers have a priori different meanings (as the Aristotelean analysis supposes), but because people entertain a hypothesis space of logical possibilities when interpreting negation markers, which context can then help disambiguate (Figure 3). When uncertain, listeners can use pragmatic reasoning to disambiguate the meaning of a double negative like not unhappy, while simultaneously showing no interpretative difference between the distinct single negations (not happy vs. unhappy) when they are heard in isolation (Figure 4, single utterances). This model makes the additional prediction, though, that when several negation markers are heard in the same context (e.g., Krifka, 2007's example above: "I don't think I was unhappy, just not happy"), that listeners can draw an interpretative difference between these two (Figure 4, multiple utterances). These inferences result from the fact that the listener has more evidence that the speaker associates different meanings for the different linguistic messages by virtue of the fact that the speaker used distinct messages in specific combinations.

Since linguistic meanings, like mental representations, are not directly observable, we

\footnotetext{
${ }^{1}$ Models are named mnemonically. Aristotle and Orwell did not defend these models themselves.
} 


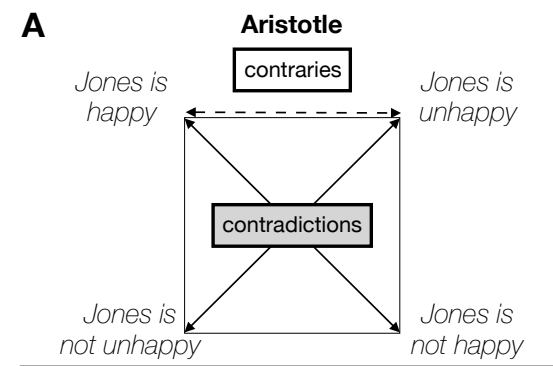

B
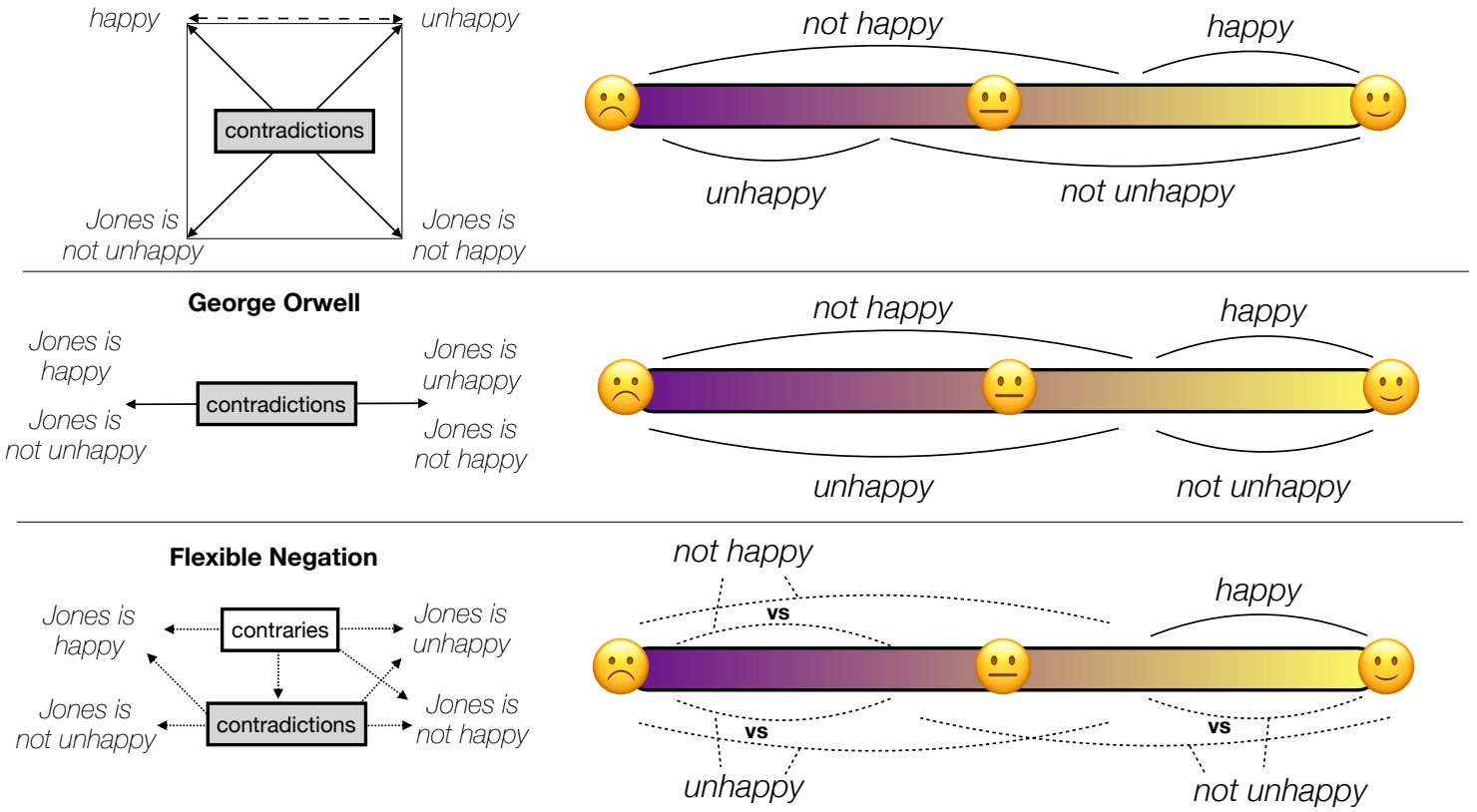

Figure 2. Space of alternative hypotheses. A: Logical relations among negation markers under three different models of meaning. B: Literal meanings of antonym quartets on a happiness scale under three different models of meaning.

formalize all hypotheses in probabilistic models of rational communication which provide the necessary linking function between these semantic hypotheses and observable behavior (Frank \& Goodman, 2014; Schöller \& Franke, 2017). The basic architecture of these models is such that a listener interprets an utterance like not unhappy as being intentionally produced by a speaker trying to communicate a degree of, say, happiness to the listener (Rational Speech Act models; Franke \& Jäger, 2015; Goodman \& Frank, 2016). The models spelled out in the SI conservatively combine previous ideas on the interpretation of gradable adjectives (Kennedy, 2007; Lassiter \& Goodman, 2017) and ambiguity in the meanings of words (Bergen, Levy, \& Goodman, 2016).

Our novel Flexible Negation hypothesis is not just an account of a few particular linguistic puzzles, but is a case study in how language users manipulate and contort language to navigate and communicate the gradations of their conceptual landscape. The general idea in the background is that listeners rationalize utterances of speakers, especially when the utterances are unexpected (Grice, 1975). A listener who hears a double negation like not unhappy looks for ways of rationalizing why the speaker has not used the simpler expression happy. The strongest form of this hypothesis is that the actual negation markers (not \& $u n$-) are irrelevant to the reasoning and interpretation; all that need occur is that a speaker use two negation markers. Thus, we investigate the dependence on linguistic form by asking how participants interpret double negatives that flagrantly use the same negation marker 


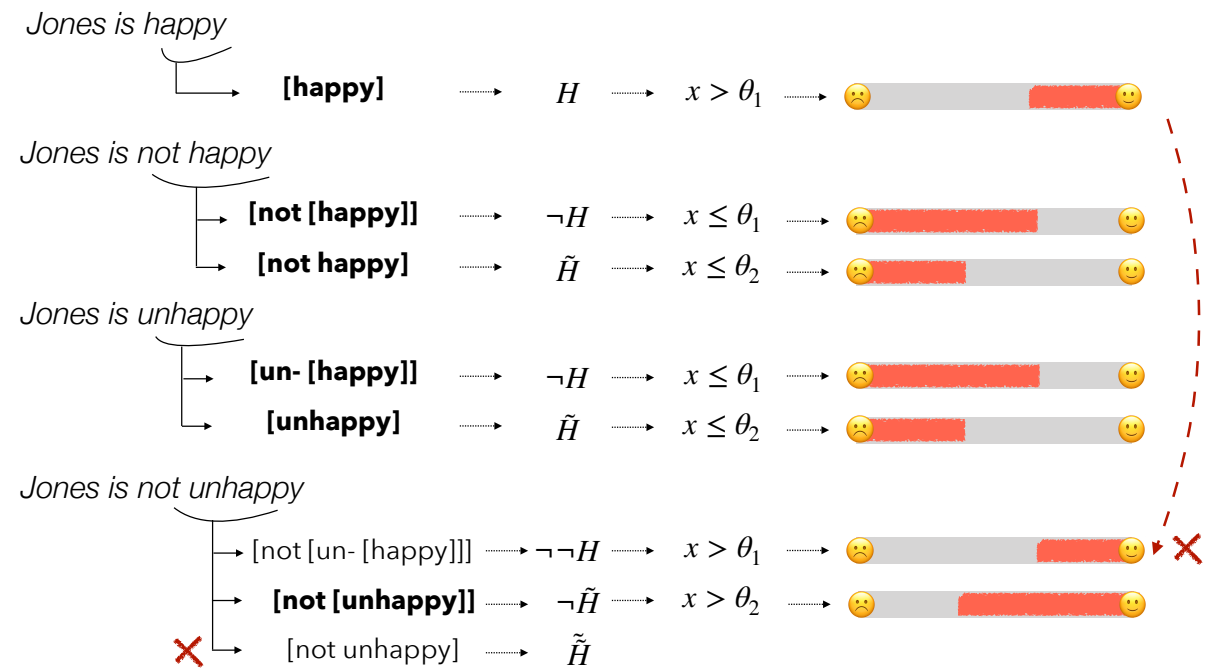

Figure 3. The Flexible Negation model reasons over a hypothesis space of possible meanings for antonym pairs and their negations. Both not happy and unhappy could signal either contradictory $\neg H$ or contrary negation $\tilde{H}$. Not unhappy can signal a double contradiction $\neg \neg H$ or a contradiction of a contrary $\neg \tilde{H}$. Contrary negation cannot take wide scope over other negation operators (see SI). A double contradiction $\neg \neg H$ is pragmatically unlikely, because the same meaning is expressed by just the simple positive $H$. Red bars denote the range of happiness values that are literally compatible with the adjective.

twice (e.g., not not happy). In order to make sense of the utterance in a principled way, listeners would have to ascribe different meanings to the two different instances of not (i.e., the utterance would indicate a contradiction of a contrary). We investigate this possibility in Experiment 3.

\section{Experiment 1: Negation in Isolation}

\section{Methods}

Participants. We recruited 120 participants from Amazon's Mechanical Turk (MTurk). This number was arrived at with the intention of getting approximately 25 ratings for each unique item in the experiment. All experiments reported here required

\begin{tabular}{lll}
\hline Adjective type & Definition & Examples \\
\hline Positive & Positive-form scalar adjective & happy, mature \\
Negated positive & "not" + positive & not happy, not mature \\
Morphological antonym & Antonym created by morphology & unhappy, immature \\
Lexical antonym & Antonym with a unique lexical item & sad, childish \\
Negated morphological antonym & "not" + morphological antonym & not unhappy, not immature \\
Negated lexical antonym & "not" + lexical antonym & not sad, not childish \\
Negated negated positive (Expt. 3) & "not" + "not" + positive & not not happy, not not mature
\end{tabular}

Table 1

Informal definitions and examples of adjective types investigated. 


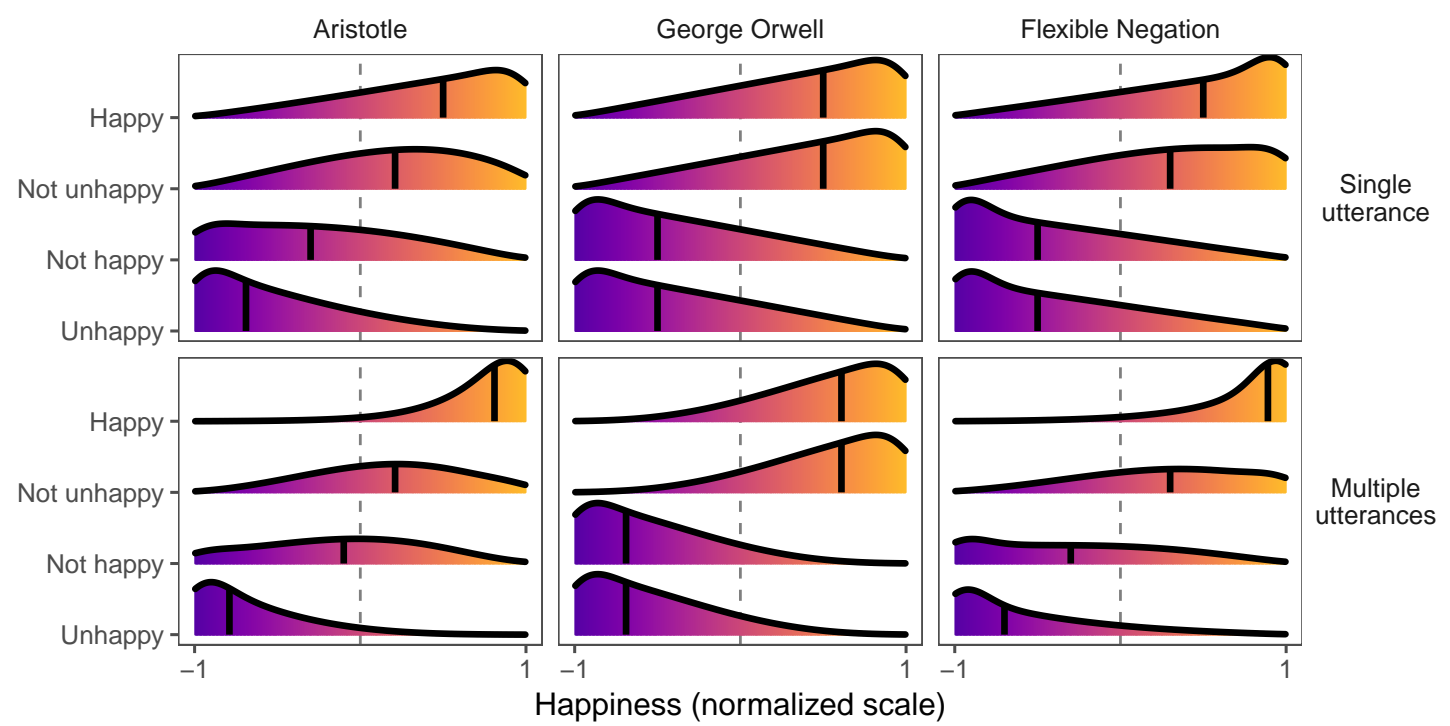

Figure 4. Model predictions for interpretations of antonym pairs and their negations under the three hypotheses. Black line shows the median of the distributions, in order to facilitate qualitative comparisons. Aristotle draws a distinction between all adjective types both when the adjective is heard in isolation (single utterance) and when adjectives are heard in the same context (multiple utterances). George Orwell never draws a meaning difference between the adjectives, even when they are heard in the same context. The Flexible Negation model a unique pattern of predictions: When adjectives are heard in isolation, the model draws no difference in meaning between not happy and unhappy but does distinguish not unhappy from happy; when the adjectives are heard in the same context, the model distinguishes among all the adjectives. Dashed line denotes the mid-point of the scale. Model predictions use minimally assumptive model parameters described in the SI.

participants with U.S. IP addresses, at least 95\% work approval rating and English as a self-reported native language. The experiment took on average 3 minutes and participants were compensated $\$ 0.40$.

Materials. We used adjectives that described properties of people. All of our adjectives were context-dependent, relative adjectives consistent with the definitions of Kennedy, 2007 and Kennedy \& McNally, 2005. We consider adjective sets consisting of four related adjective types (see Table 2): positives (e.g., happy, tall), antonyms (e.g., short, unhappy), and their respective negations (not X). 20 adjective sets were constructed, 10 for lexical antonyms (short) and 10 for morphological antonyms (unhappy).

Procedure. On each trial, participants read a statement introducing a person using a gradable adjective of one of four adjective types: positives (e.g., happy, tall), antonyms (e.g., short, unhappy), and their respective negations (not $\mathrm{X}$ ). Antonyms were one of two types: morphological (e.g., unhappy) and lexical (e.g., short). Participants rated the character on a scale from "the most positive person" to "the most antonym person" (item-dependent) using a slider bar (Fig. 5A). Participants rated one sentence at a time and saw items from both antonym types throughout the experiment. Each participant completed a total of 16 trials, 
Table 2

\begin{tabular}{ll}
\hline Morphological antonyms & Lexical antonyms \\
\hline attractive, unattractive & beautiful, ugly \\
educated, uneducated & brave, cowardly \\
friendly, unfriendly & fat, skinny \\
happy, unhappy & hard-working, lazy \\
honest, dishonest & loud, quiet \\
intelligent, unintelligent & proud, humble \\
interesting, uninteresting & rich, poor \\
mature, immature & strong, weak \\
polite, impolite & tall, short \\
successful, unsuccessful & wise, foolish \\
\hline
\end{tabular}

Items in Experiment 1.

with exactly 2 repetitions of each adjective type for each antonym type. No participant saw two instances from the same adjective set.

\section{Results}

6 participants were excluded for self-reporting a native language other than English, leaving a remainder of 114 participants for these analyses, which resulted in on average 23 ratings for each unique adjective in our stimulus set. The qualitative predictions of our models concern the ordering within a set of alternatives for different antonym types

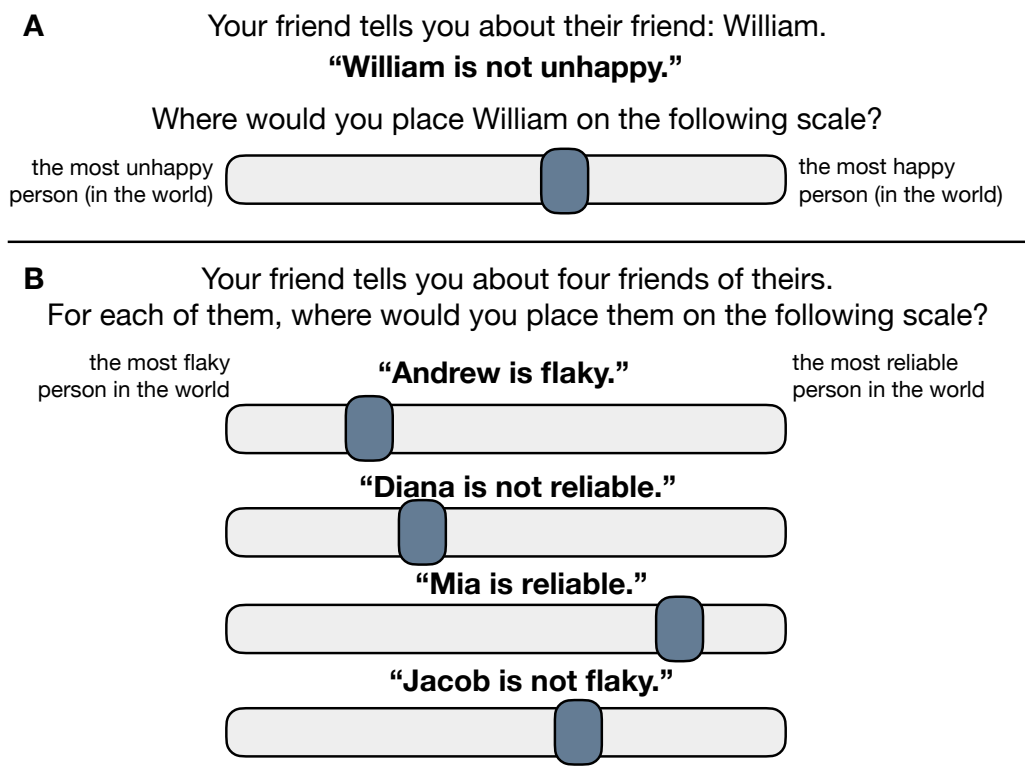

Figure 5. Example experimental trials for (A) single utterance (Expts. 1, 2) and (B) multiple utterances (Expts. 2,3) conditions. "in the world" wording for endpoints was used in Expts. 2 \& 3. (A) shows a trial from a morphological antonym set while (B) shows a lexical antonym set. 


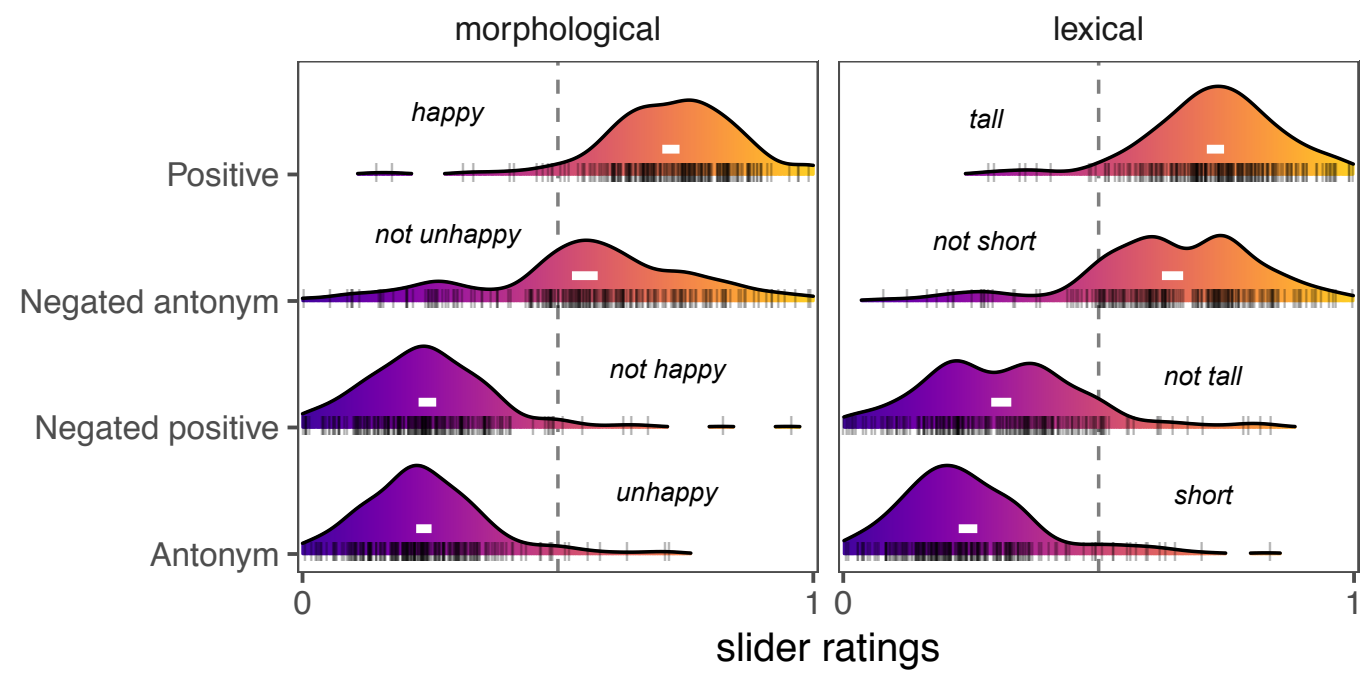

Figure 6. Experiment 1 results. Empirical distributions of responses for adjective sets with morphological antonyms (e.g., "unhappy") and lexical antonyms (e.g., "sad"). Dashed line indicates the midpoint of the scale. Dashes below density plots denote individual responses. White bars denote bootstrapped $95 \%$ confidence intervals for the means.

(morphological vs. lexical). Figure 6 shows the empirical distributions for each of the four adjective types for both morphological and lexical antonyms adjective sets. Critically, as predicted by the Flexible Negation model, adjective sets with morphological antonyms show only a partial ordering, with negated positives (e.g., not happy) and morphological antonyms (e.g., unhappy) receiving the same ratings; at the same time, the adjective sets with with lexical antonyms show a full ordering (i.e., all adjective types receive distinct interpretations).

To confirm these observations, we built a linear mixed model predicting the raw ratings in terms of fixed effects of antonym type (morphological vs. lexical; dummy-coded with morphological default level), adjective type (forward-difference coded in order: antonym, negated positive, negated antonym, positive $)^{2}$, and their interaction; the model also included random intercepts and random slopes of adjective type by-participant and by-item. ${ }^{3}$ Consistent with our observations, the difference between the antonym vs. negated positive levels of adjective type interacted significantly with antonym type (morphological vs. lexical; $\beta=-0.059$, $\mathrm{t}(16)=-2.40, p=0.029)$ such that the difference between negated positives and antonyms was stronger for lexical antonyms than morphological antonyms. At the same time, the negated positive vs. antonyms contrast was not significantly different for the morphological antonyms (e.g., unhappy vs. not happy; $\beta=-0.007, \mathrm{t}(17)=-0.39, p=0.701$ ). Running the same model with the antonym type coded with lexical antonyms as the default level reveals that the negated positive vs. antonyms contrast was significantly different for the

\footnotetext{
${ }^{2}$ Forward difference coding compares adjacent-levels of a factor, which allows for the following comparisons: antonym vs. negated positive, negated positive vs. negated antonym, negated antonym vs. positive.

${ }^{3}$ This, and all subsequent regression models, were the maximal mixed-effects model that converged for the data set that additionally explained significantly more variance than models with simpler mixed-effects structures, using the lme4 package in R (Bates, Mächler, Bolker, \& Walker, 2015).
} 
lexical antonyms (e.g., short vs. not tall; $\beta=-0.065, \mathrm{t}(17)=-3.75, p=0.002$ ).

We observe further that negated morphological antonyms (e.g., not unhappy) were rated differently and lower than positives (e.g., happy; $\beta=-0.170, \mathrm{t}(25)=-9.50, p<0.001$ ). We additionally observe that negated morphological antonyms (e.g., not unhappy) were rated overall lower than negated lexical antonyms (e.g., not tall; Figure 6), which manifested as an interaction in the negated antonym vs. positive contrast with the lexical vs. morphological items $(\beta=0.081, \mathrm{t}(19)=3.46, p=0.003)$. Negated antonyms received a distinct bimodal distribution wherein most ratings were slightly positive but a clearly distinguishable minority distribution of ratings were slightly negative (e.g., not dishonest meaning not honest). This weakly negative interpretation for negated antonyms was present at least somewhat in every item and in most participants. This interpretation may be the result of participants attributing politeness to the speaker. In fact, when speakers care about a listener's self-image, human participants tend to endorse indirect utterances more: Not dishonest may be an indirect way of saying that a person is not honest (Yoon, Tessler, Goodman, \& Frank, 2017).

While we note the differences between lexical and morphological antonyms in this experiment, the direct comparison of these two kinds of adjectives is difficult. We have coded the antonyms in the morphological sets as positive and negative (or, antonym) by appealing to the morphology (e.g., happy is the positive adjective, while unhappy is the antonym); a similar assignment of the lexical antonyms to positive and negative is not always possible. Some pairs have a clear unmarked form: tall is the positive adjective because when describing the height of a person, we say six feet tall and not six feet short. For items that did not have a clear unmarked form (e.g., fat and skinny), we assigned the adjective that conveyed a greater amount to be the positive (i.e., fat conveys more weight than skinny); thus, the positive adjective is not necessarily the socially more desirable feature. ${ }^{4}$ Because of these differences between lexical and morphological antonym sets, we treated this experiment as exploratory and curated a more tightly controlled set of materials for Experiments $2 \& 3$.

\section{Experiment 2: Negation with Implicit Contrasts}

Expt. 1 revealed an asymmetry: Lexical antonyms (e.g., short) were clearly distinguished from negated positives (e.g., not tall), whereas morphological antonyms were not (e.g., unhappy $\approx$ not happy). In Expt. 1, our adjective sets varied both in terms of their antonym type (morphological vs. lexical) as well as the actual degree scales being described (e.g., height for tall/short vs. happiness for happy/unhappy). Many adjective sets have both morphological and lexical antonyms (e.g., happy/unhappy/sad). Here, we aim to replicate the previous findings using adjectives that describe the same semantic scales. Also, we test

\footnotetext{
${ }^{4}$ In addition, in the empirical data, we see a bimodal distribution of responses for the negated lexical antonyms and negated positives; thus, one may be concerned that this bimodal distribution is the result of an improper assignment of lexical antonyms to either the positive or antonym form (e.g., skinny is actually the positive adjective and fat is the antonym). If this were so, we would expect this bi-modality to occur across the items but not within items; however, when we look at the item-specific distributions of responses, we do not see clear evidence for this, but rather we see the bi-modality occurring within several items from the lexical sets (see SI).
} 
our second prediction that hearing multiple utterances in the same context will produce the full ordering for morphological antonym sets (Fig. 4).

\section{Methods}

Participants. We recruited 750 participants from MTurk. The experiment comprised four between-subjects experimental conditions arranged in a $2 \mathrm{x} 2$ design: antonym type (morphological vs. lexical) X context (single vs. multiple utterances). 300 participants were assigned to each antonym type in the single utterance contexts, and 75 participants were assigned to each in the multiple utterances conditions. These numbers follow from the intention of getting approximately 45 ratings for each unique adjective in the experiment. The single utterance task took on average 3 minutes and participants were compensated $\$ 0.40$; multiple utterances took on average 5 minutes and participants were compensated $\$ 0.80$. Exclusion criterion, sample size, procedure, and the analysis described below were preregistered: osf.io/p7f25/.

Materials. To best isolate the contribution of morphological vs. lexical antonyms, we curated adjective sets consisting of words for properties of people, such that both types of antonyms existed for the same positive adjective (e.g., happy $\rightarrow$ unhappy, sad; Table 4). Lexical antonyms were selected from a set of possibilities produced from a small survey $(\mathrm{n}=18)$ on MTurk eliciting "opposites" for a list of 30 positive-form adjectives which had morphological antonyms. In this antonym elicitation, participants saw the same material as in the main experiment (e.g., "Your friend tells you about their friend: William. William is forgiving.") and asked "What is the opposite of adjective?" (e.g., "What is the opposite of forgiving?"). From the list of freely-produced opposites, the first author chose the one that intuitively best conveyed the same scalar dimension as the morphological antonym and which was not already used as a lexical antonym for another item (e.g., opposite of forgiving $\rightarrow$ resentful; opposite of kind $\rightarrow$ cruel, because opposite of friendly $\rightarrow$ mean). Ten out of the original 30 items were dropped for either not having such a well-suited lexical antonym (e.g., moral) or for having a well-suited lexical antonym that conflicted with another item (e.g., compassionate $\rightarrow$ cold, but also affectionate $\rightarrow$ cold $)$.

Procedure. In the multiple utterances conditions, participants rated all four adjective types simultaneously, each referring to a different person (Fig. 5B), for a total of 12 trials. The single utterances conditions were similar to that of Expt.1: Participants rated one sentence at a time (e.g., "Greg is not unhappy"), each from a unique adjective set (e.g., never rated both unhappy and not happy), completing a total of 12 trials, with exactly 3 repetitions of each adjective type (positive, antonym, and their negations). In contrast to Expt. 1, antonym type (morphological vs. lexical) was a between-participants factor. In addition, the slider bar endpoints were relabeled to "the most \{positive, negative $\}$ person in the world"; without "in the world", there is a salient interpretation of the endpoints indicating "the most \{positive, negative $\}$ person (of these four)" in the multiple utterances conditions. 


\begin{tabular}{lll}
\hline Positive adjective & Morphological antonym & Lexical antonym \\
\hline affectionate & unaffectionate & cold \\
ambitious & unambitious & lazy \\
attractive & unattractive & ugly \\
educated & uneducated & ignorant \\
forgiving & unforgiving & resentful \\
friendly & unfriendly & mean \\
generous & ungenerous & stingy \\
happy & unhappy & sad \\
honest & dishonest & deceitful \\
intelligent & unintelligent & stupid \\
interesting & uninteresting & boring \\
kind & unkind & cruel \\
mature & immature & childish \\
patriotic & unpatriotic & traitorous \\
polite & impolite & rude \\
rational & irrational & crazy \\
reliable & unreliable & flaky \\
resourceful & unresourceful & wasteful \\
sincere & insincere & fake \\
tolerant & intolerant & bigoted \\
\hline
\end{tabular}

Table 3

Items used in Experiment 2.

\section{Results}

35 participants were excluded for self-reporting a native language other than English, leaving 715 participants for these analyses. Results for each adjective type in each condition are shown in Fig. 7.

As we did in Expt.1, we evaluated our hypothesis that, in the single utterance condition, morphological antonyms behave like the Flexible Negation model (i.e., show a partial ordering) while lexical antonyms show a true ordering (like bonafide contraries). We considered data only from the single utterances conditions and built a linear mixed model predicting the raw ratings in terms of antonym type (morphological vs. lexical; dummycoded with morphological as the base case), adjective type (forward-difference coded in order: antonym, negated positive, negated antonym, positive) and their interaction; the model also included random intercepts and random slopes of adjective type by-participant and by-item. Consistent with our hypothesis, the antonym vs. negated positive difference was greater for lexical antonyms than morphological antonyms (i.e., an adjective type by antonym type interaction; $\beta=-0.021, \mathrm{t}(565)=-2.68, p=0.008)$. Further, the antonym vs. negated positive difference for morphological antonyms (e.g., unhappy vs. not happy) was not significantly different from zero $\left(\beta=-6.354 \cdot 10^{-4}, \mathrm{t}(49)=-0.10, p=0.920\right)$. Running the same model with the antonym type coded with lexical antonyms as the default level reveals that the antonym vs. negated positive contrast was significantly different for the lexical antonyms (e.g., sad vs. not happy; $\beta=-0.022$, t $(51)=-3.45, p=0.001$ ).

Our second main hypothesis was that context (single vs. multiple utterances) modulates the interpretive difference between morphological antonyms and negated positives. Specifically, we predict that morphological antonyms will be interpreted more negatively than negated positives in a context with multiple adjectival utterances. To evaluate this 


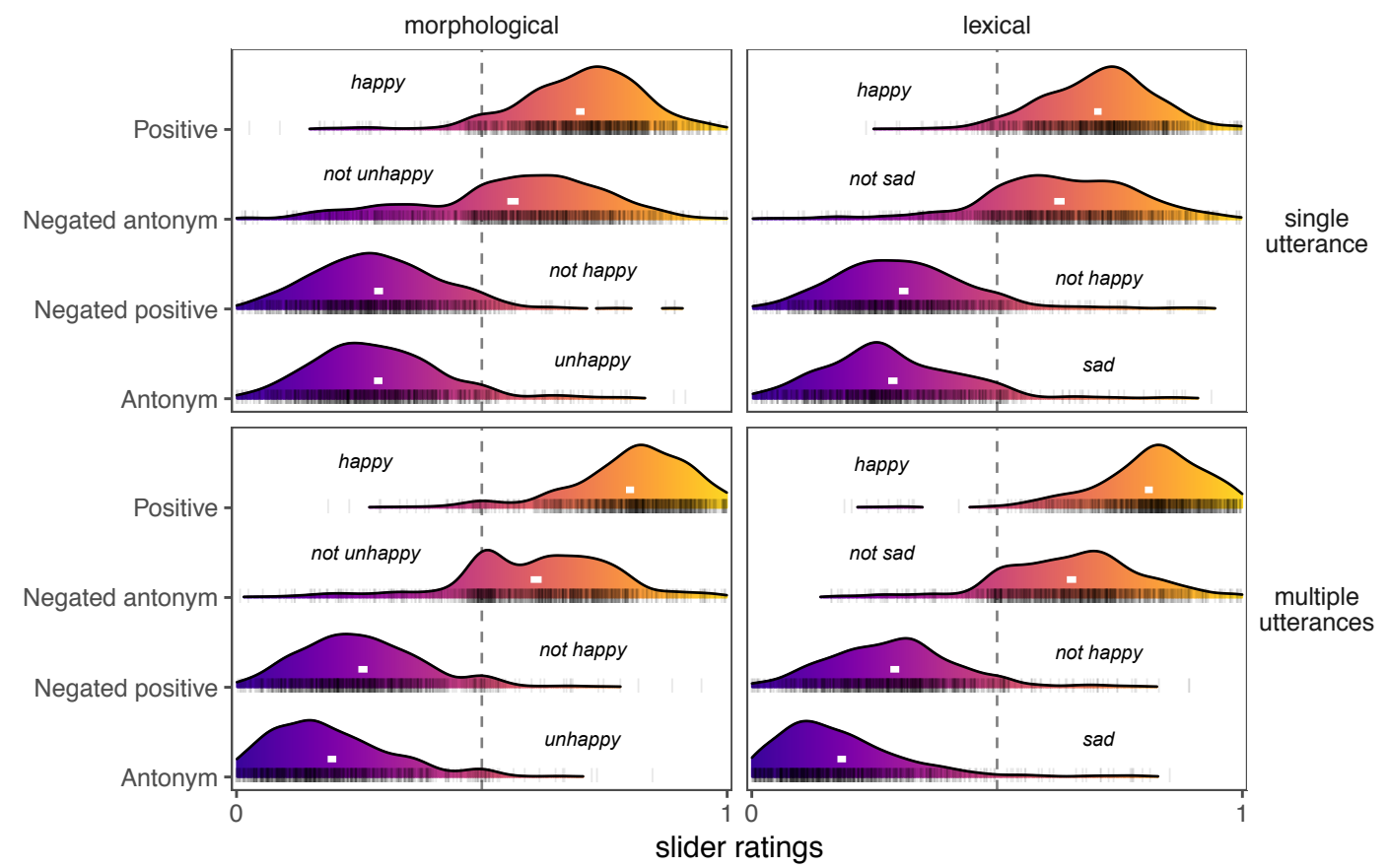

Figure 7. Experiment 2 results. Empirical distributions of responses for adjective sets with morphological antonyms (e.g., "unhappy") and lexical antonyms (e.g., "sad") for the single utterance and multiple utterances conditions. Dashed line indicates the midpoint of the scale. Dashes below density plots denote individual responses. Width of white rectangles denotes bootstrapped $95 \%$ confidence intervals for the means.

hypothesis, we considered data only from the morphological antonyms conditions and built a linear mixed model predicting the raw ratings in terms of adjective type (forward-difference coded, as above), context (single vs. multiple utterances; multiple as base case) and their interaction; the model also included random intercepts and random slopes of adjective type by-participant and by-item. The antonym vs. negated positive difference was significantly greater in the multiple utterances condition than the single utterance condition (i.e., an adjective type by context interaction; $\beta=0.063$, $\mathrm{t}(6457)=6.73, p<0.001)$, such that antonyms were interpreted more negatively than negated positives in the multiple utterances condition $(\beta=-0.064, \mathrm{t}(268)=-9.27, p<0.001)$, but not in the single utterance condition (same contrast as previous paragraph).

The predictions of the Flexible Negation model are ambiguous about the relevant three-way interaction (antonym vs. negated positive by lexical vs. morphological adjective type by context). On the one hand, for the antonym vs. negated positive contrast, the model predicts no difference in meaning for morphological antonyms when presented in isolation, but does predict meaning differences when the alternatives are presented together, whereas the difference is expected to occur for lexical antonyms in both context conditions. On the other hand, the model's inferences about the likely meaning of all of the adjectives gets further differentiated as a result of being presented in the same context (i.e., all adjectives get more specific interpretations). Thus, it is not clear a priori that the Flexible Negation 
model predicts a three-way interaction nor the direction of the interaction. As an exploratory analysis, we examined these effects in a full three-way interactive model and found the relevant three-way interaction was in the direction of lexical antonyms showing a larger antonym vs. negated positive difference in the multiple utterance condition; this effect was not significant, though $(\beta=0.012, \mathrm{t}(469)=1.64, p=0.102)$.

\section{Experiment 3: Flagrant Double Negatives}

In this paper, we propose that the logic of negated morphological antonyms (not unhappy) proceeds via a listener reasoning about why a speaker used two distinct negation markers (not \& un-) and concluding that the speaker must have had different meanings in mind (contradiction and contrary, respectively) for the different negation markers. Yet, the production of a double negative doesn't require distinct negation markers: One can grammatically produce a double negative flagrantly using the same negation marker twice (e.g., not not happy). ${ }^{5}$ Is the inferential cognitive mechanism that produces meanings for negated antonyms like not unhappy sufficiently flexible to accommodate double negations that rely upon the iterative application of the same logical word (not not)? If so, then we would expect flagrant double negatives like not not happy to be interpreted in a way similar to more canonical double negatives like not unhappy. Such a finding would suggest that communicative reasoning can contort one of the basic logical structures in language. In this experiment, we investigate interpretations of double "not" constructions using the multiple utterances context from Experiment 2.

\section{Methods}

Participants. We recruited 75 participants from MTurk to match the sample size of the same condition in Expt. 2. These numbers follow from the intention of getting approximately 45 ratings for each unique adjective in the experiment. The experiment took on average 5 minutes and participants were compensated $\$ 0.90$. Exclusion criterion, sample size, procedure, and the analysis described below were preregistered: osf.io/vjhak.

Materials and procedure. The materials and procedure were identical to that of the multiple utterances condition of Expt. 2. The main difference in this experiment is that participants are presented with the following alternatives: positives, negated positives, morphological antonyms, and negated negated positives (e.g., happy, not happy, unhappy, not not happy). This experiment was conducted eighteen months after Expt. 2, and due to concerns about recently declining data quality on MTurk, this experiment included an additional memory check wherein participants had to select all of the items that they recall being tested on from a list of 10 items ( 5 real and 5 distractor). We pre-registered the exclusion criterion of removing participants who failed to respond correctly to at least 7 of the 10 items.

\footnotetext{
${ }^{5}$ While the usage of the same negation marker twice (e.g., not not) is not common, examples are easy to find on webpages and news articles, suggesting that speakers use this construction to achieve real communicative goals.
} 


\section{Results}

All participants self-reported only English as their native language. 5 participants were excluded for failing to respond correctly to at least 7 of the 10 memory check items, leaving 40 participants for these analyses. Results for each adjective type are shown in Figure 8.

Our main hypothesis concerns the interpretation of flagrant double negative statements that use the same negation marker twice (not not happy). We built a mixed-effects regression model with random by-participant and by-item intercepts and slopes (where the slopes refer to the effect of the adjective type, forward-difference coded as before: happy vs. not happy vs. not not happy vs. unhappy). As is visually apparent, the two negatives did not simply cancel to make a positive: not not happy received a significantly more negative interpretation than happy $(\beta=-0.281, \mathrm{t}(40)=-8.76, p<0.001)$. This suggests that participants actively try to make sense of seemingly redundant, contradictory information in a way that would be informative for a speaker to produce. In addition, the result from Experiment 2 (multiple utterances condition) replicated for the morphological antonyms: not happy and unhappy were differentiated in meaning $(\beta=-0.040, \mathrm{t}(40)=-3.33, p=0.002)$. Contrary to our hypothesis, however, we did not find evidence that the flagrant double negative not not happy received on-average a positive interpretation $(\beta=0.036, \mathrm{t}(39)=1.07, p=0.29)$; inspection of the fitted random-effects suggested this was due to large subject-wise variation in the interpretation of these flagrant double negatives. We return to this observation in the General Discussion.

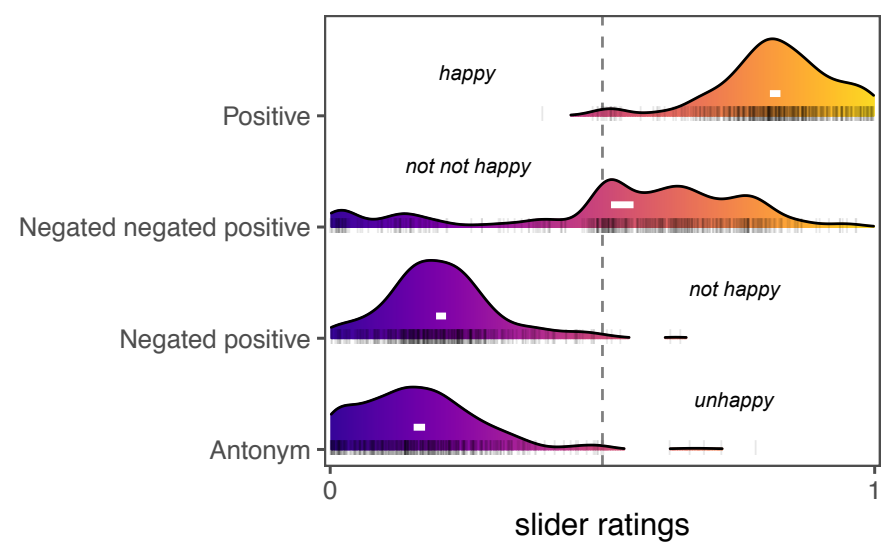

Figure 8. Experiment 3 results. Dashed line indicates the midpoint of the scale. Dashes below density plots denote individual responses. White bars denote bootstrapped $95 \%$ confidence intervals for the means.

\section{General Discussion}

This paper provides a computational solution to an outstanding puzzle in natural language understanding: How to interpret double negatives (e.g., not unhappy; Horn, 1991; Krifka, 2007; Rett, 2014). We additionally discovered and confirmed a surprising 
empirical result: unhappy (morphological antonyms) and not happy (negated positives) are interpreted identically, except when presented in the same context. Our model that represents flexibility in how to interpret overt negation markers (un-, not) predicts this very result, while alternative models that treat negation markers as only carrying a single possible meaning fall short.

It is noteworthy that we are able to recover, both in our model and empirically, the ordering predicted by Krifka (2007) for morphological antonyms (unhappy < not happy $<$ not unhappy < happy), when a listener hears multiple adjectival utterances in the same context (Experiment 2; multiple utterances condition). Other recent accounts derive similar predictions based on quite different kinds of assumptions (Krifka, 2007; Rett, 2014; Cable, 2018), but only our model makes predictions about the context-dependence of these inferences. This work thus carries with it an account of a robust linguistic intuition: Potentially equivalent utterances receive differential interpretations when produced by the same speaker in close proximity, an instance of mutual exclusivity reasoning (Markman, 1989). In Experiment 3, we discovered that this inference goes beyond using distinct expressions for negation: Double negative statements that use the same negation marker twice (e.g., not not happy) are interpreted in a similar way to negated morphological antonyms (e.g., not unhappy), indicating the underlying cognitive mechanism used to make sense of seemingly redundant, contradictory content (not not) is guided by a principled underlying logic: Two negatives can be coherently combined if one is a contradiction and the other is a contrary. This result also suggests a strong view of flexible communication with negation: The same negation marker can be interpreted in two different ways within a single utterance.

Our work builds upon previous studies on the meanings of negated adjectives and negated antonyms with our straight-forward response measurements and comparisons to other adjective forms in different presentational contexts. We've extended earlier work showing that negated lexical antonyms (e.g., not $d u l l$ ) are distinct from positive adjectives (e.g., sharp) when presented simultaneously (Giora, Balaban, Fein, \& Alkabets, 2005) to both the case of morphological antonyms (e.g., not unhappy) and when presented in isolation (single utterance conditions). We also provide convergent evidence that supports the phenomenon of negative strengthening or inference towards the antonym, where negating an adjective (e.g., not intelligent) can lead to the stronger interpretation than literally implied (e.g., rather stupid; Ruytenbeek, Verheyen, \& Spector, 2017; Gotzner, Solt, \& Benz, 2018).

Our formalization of lexical uncertainty about the meaning of natural language negation adds to a growing movement to treat the combinatorial rules of grammar as not totally separable from the lexicon (e.g., Bybee, 2006; O'Donnell, 2015). Recent psycholinguistic evidence supports the idea that utterances which are heavily used will be processed as unique lexical entries while less frequent phrases will be understood compositionally (Morgan \& Levy, 2016). The two types of negation meaning we considered - contrary and contradictory opposition - can be seen as a lexicalized form of opposition and a compositional rule, respectively. ${ }^{6}$ In our modeling, we assumed all logically possible meanings for negation were

\footnotetext{
${ }^{6}$ Contraries act as lexicalized form of negation because their meanings refer to semantic variables distinct from the positive adjective (in particular, a different threshold; see SI), whereas contradictions refer to the same semantic variable as the positive adjective.
} 
equally likely a priori: A further test of our negation uncertainty model would be to see if usage frequency can serve as a proxy for this prior over lexica.

In this paper, we aimed to explain the modal interpretations of antonym pairs and their negations, but our empirical data suggest more nuance and variability in judgments, even in our minimal paradigms. While the modal response for negated antonyms was indicating a slightly positive state, there were consistent responses indicating a slightly negative interpretation as well (e.g., the meaning expressed by "he's not [un]reliable but he is kind of flaky", with prosodic focus on [un-]). This interpretation may be the result of participants attributing politeness to the speaker: Not unreliable may be an indirect way of saying that a person is not reliable (Yoon et al., 2017). We also see a very slight hint of strongly negative interpretations (e.g., very unhappy) from the flagrant double negatives of Expt. 3 (not not happy): The additivity of negations, or negative concord, is not often associated with standard English, though it is relatively common cross-linguistically (e.g., in Italian: non capisco niente, literal translation: I don't understand nothing; Zeijlstra, 2004) including in African American Vernacular English (e.g., Mohammad Ali: "Ain't never been another fighter like me"; Labov, 1972; Howe, 2005). Finally, though we did not find any evidence of double negatives being interpreted as strong positives in the minimal contexts used in our experiments, double negatives are employed in understatement (Bolinger, 1972): In the appropriate context, "I was not unaware of the problem" could mean I was damn well aware of the problem. Future work should investigate the relevant context and prosodic cues that can be used to derive diverse interpretations from antonym pairs and their negations. Additionally, though our experiments are conducted in English, we expect these patterns to hold cross-linguistically for languages without negative concord and with the negation markers that can act as productively as English not and un-.

The fact that the meaning of explicit negation markers like not and un- is flexible allows for an ambiguity that can be exploited by speakers. Our results suggest that if a speaker claims an individual is "not not innocent", the speaker could simultaneously convey that the person is innocent as well as that they are at least somewhat guilty. Such an obfuscation can lead to confusion within listeners or divergent beliefs across listeners, with some people believing in innocence and others in guilt as a kind of "dog whistle". It may as well impose gradability (e.g., degrees of guilt) onto what would typically be a binary distinction (i.e., innocent or guilty). Understanding the psychological reality of this ambiguity will help us navigate the consequences of the plausibly deniable misinformation that lays in the language of negation. 
References

Bardwick, J. M. (1986). The plateauing trap: How to avoid it in your career... and your life. American Management Association.

Bates, D., Mächler, M., Bolker, B., \& Walker, S. (2015). Fitting linear mixed-effects models using lme4. Journal of Statistical Software, 67(1), 1-48.

Bergen, L., Levy, R., \& Goodman, N. D. (2016). Pragmatic reasoning through semantic inference. Semantics and Pragmatics, 9.

Blutner, R. (2004). Pragmatics and the lexicon. Handbook of pragmatics, 488-514.

Bolinger, D. (1972). Degree words. Mouton and Company.

Bybee, J. L. (2006). From usage to grammar: The mind's response to repetition. Language, 82(4), 711-733.

Cable, S. (2018). The good, the 'not good', and the 'not pretty': Negation in the negative predicates of tlingit. Natural Language Semantics, 26 (3-4), 281-335.

Frank, M. C., \& Goodman, N. D. (2014). Inferring word meanings by assuming that speakers are informative. Cognitive Psychology, 75, 80-96.

Franke, M., \& Jäger, G. (2015). Probabilistic pragmatics, or why Bayes' rule is probably important for pragmatics. In Zeitschrift für sprachwissenschaft (pp. 3-44).

Giora, R., Balaban, N., Fein, O., \& Alkabets, I. (2005). Negation as positivity in disguise. Figurative language comprehension: Social and cultural influences, 233-258.

Goodman, N. D., \& Frank, M. C. (2016). Pragmatic language interpretation as probabilistic inference. Trends in Cognitive Sciences, 20(11), 818-829.

Gotzner, N., Solt, S., \& Benz, A. (2018). Scalar diversity, negative strengthening, and adjectival semantics. Frontiers in Psychology, 9(SEP), 1-13. doi: 10.3389/fpsyg.2018.01659

Grice, H. P. (1975). Logic and Conversation. In Syntax and semantics 3: Speech acts (pp. 41-58).

Herbert, C., Deutsch, R., Sütterlin, S., Kübler, A., \& Pauli, P. (2011). Negation as a means for emotion regulation? Startle reflex modulation during processing of negated emotional words. Cognitive, Affective and Behavioral Neuroscience, 11(2), 199-206. doi: 10.3758/s13415-011-0026-1

Horn, L. R. (1989). A natural history of negation. University of Chicago Press.

Horn, L. R. (1991). Duplex negatio affirmat...: The economy of double negation. CLS 27-II: Papers from the parasession on negation, 80-106.

Howe, D. (2005). Negation in African American Vernacular English. Aspects of English negation, 132, 173.

Jespersen, O. (1917). Negation in English and other languages. Kobenhavn: Host.

Jespersen, O. (1924). The philosophy of grammar. London: Allen \& Unwin.

Kennedy, C. (2007). Vagueness and grammar: the semantics of relative and absolute gradable adjectives. Linguistics and Philosophy, 30, 1-35.

Kennedy, C., \& McNally, L. (2005). Scale structure, degree modification, and the semantics of gradable predicates. Language, 345-381.

Krifka, M. (2007). Negated antonyms: Creating and filling the gap. Presupposition and Implicature in Compositional Semantics, 163-177.

Labov, W. (1972). Negative attraction and negative concord in English grammar. Language, $773-818$. 
Lakoff, G. (2008). Women, fire, and dangerous things. University of Chicago press.

Lassiter, D., \& Goodman, N. D. (2017). Adjectival vagueness in a Bayesian model of interpretation. Synthese.

Lindquist, K. A., Satpute, A. B., \& Gendron, M. (2015). Does language do more than communicate emotion? Current Directions in Psychological Science, 24(2), 99-108. doi: $10.1177 / 0963721414553440$

Markman, E. M. (1989). Categorization and naming in children: Problems of induction. MIT Press.

Morgan, E., \& Levy, R. (2016). Abstract knowledge versus direct experience in processing of binomial expressions. Cognition, 157, 384-402.

O'Donnell, T. J. (2015). Productivity and reuse in language: A theory of linguistic computation and storage. MIT Press.

Orwell, G. (1946). Politics and the English language. Horizon.

Rett, J. (2014). The semantics of evaluativity. Oxford University Press.

Ruytenbeek, N., Verheyen, S., \& Spector, B. (2017). Asymmetric inference towards the antonym: Experiments into the polarity and morphology of negated adjectives. Glossa: a journal of general linguistics, 2(1), 92. doi: 10.5334/gjgl.151

Sapir, E. (1944). Grading, a study in semantics. Philosophy of science, 11(2), 93-116.

Satpute, A. B., Nook, E. C., Narayanan, S., Shu, J., Weber, J., \& Ochsner, K. N. (2016). Emotions in "black and white" or shades of gray? how we think about emotion shapes our perception and neural representation of emotion. Psychological Science, 27(11), 1428-1442. doi: $10.1177 / 0956797616661555$

Schöller, A., \& Franke, M. (2017). Semantic values as latent parameters: Testing a fixed threshold hypothesis for cardinal readings of few \& many. Linguistic Vanguard, 3(1).

Tiersma, P. M. (1999). Legal language. University of Chicago Press.

Yoon, E. J., Tessler, M. H., Goodman, N. D., \& Frank, M. C. (2017). "I won't lie, it wasn't amazing": Modeling polite indirect speech. In Proceedings of the 39th Annual Meeting of the Cognitive Science Society.

Zeijlstra, H. (2004). Sentential negation and negative concord. Netherlands Graduate School of Linguistics. 